\title{
Interaction of maize Opaque-2 and the transcriptional co-activators GCN5 and ADA2, in the modulation of transcriptional activity
}

\author{
Riyaz A. Bhat ${ }^{1}$, Jan W. Borst ${ }^{2}$, Marcus Riehl ${ }^{1,3}$ and Richard D. Thompson ${ }^{1,4, *}$ \\ ${ }^{1}$ Max Planck Institute for Plant Breeding Research, Carl-von-Linné Weg 10, D-50829 Cologne, Germany; \\ ${ }^{2}$ MicroSpectroscopy Centre, Wageningen University, Dreyenlaan 3, 6703 HA Wageningen, The Netherlands; \\ ${ }^{3}$ Present addresses: Novoplant, D-06466 Gatersleben, Germany; ${ }^{4}$ INRA Research Unit for genetics and \\ ecophysiology of grain legumes (URLEG), BP 86510, 21065 Dijon Cédex, France (*author for correspon- \\ dence; e-mail thompson@epoisses.inra.fr)
}

Received 2 March 2004; accepted in revised form 7 June 2004

Key words: acceptor photobleaching, co-activators, FLIM, FRET, transcriptional activation

\begin{abstract}
Maize Opaque-2 ( $\mathrm{ZmO} 2)$, a bZip class transcription factor has been shown to activate the transcription of a series of genes expressed in the maturation phase of endosperm development. Activation requires the presence of one or more enhancer binding sites, which confer the propensity for activation by $\mathrm{ZmO} 2$ on heterologous promoters and in heterologous plant cell types, such as tobacco mesophyll protoplasts. The region of $\mathrm{ZmO} 2$ required for conferring transcriptional activation has been localised to a stretch of acidic residues in the $\mathrm{N}$-terminal portion of the $\mathrm{ZmO} 2$ sequence, which is conserved between O2-related bZip factor sequences. Previously we identified the maize homologues of yeast transcriptional co-activators GCN5 and ADA2 that are implicated in nucleosome modification and transcription. In the present study we have shown that transcriptional modulation by $\mathrm{ZmO} 2$ involves the intranuclear interaction of $\mathrm{ZmO} 2$ with ZmADA2 and ZmGCN5. Förster resonance energy transfer (FRET) based techniques have enabled us to estimate the intracellular site of these intermolecular interactions. As a functional readout of these intranuclear interactions, we used the $\mathrm{ZmO} 2$ responsive maize $\mathrm{b}-32$ promoter to drive the $\beta$-glucuronidase (GUS) in the presence and absence of ZmGCN5 and ZmADA2. Our results suggest that the likely recruitment of ZmADA2 and ZmGCN5 modulates the transactivation of b-32 promoter by $\mathrm{ZmO} 2$ and that there may be a competition between $\mathrm{ZmGCN} 5$ and $\mathrm{ZmO} 2$ for binding to the amino-terminal of ZmADA2. The results may be taken as a paradigm for other processes of transcriptional modulation in planta involving acidic activation domains.
\end{abstract}

\section{Introduction}

The eukaryotic transcription initiation machinery, consisting of RNA polymerase II and other additional components is recruited to the promoters of target genes by transcriptional enhancers or activators (Holstege et al., 1998). Specific activator proteins bind at one or more locations upstream of the TATA sequence (Guarente et al., 1982; Drysdale et al., 1995). Activators can interact directly with one of the basic transcription factors (Lin et al., 1991; Geisberg et al., 1994). However there are indications that they interact with basic factors through co-activators (Drysdale et al., 1995). These co-activators may also modify nucleosomes to facilitate access by the transcription machinery. Yeast GCN4, a bZip type transcriptional activator, has been shown to interact with the GCN5-ADA2 co-activator complex implicated in nucleosome modification (Barlev et al., 1995). GCN4 binds to DNA as a homodimer and activates genes encoding amino acid biosynthesis enzymes in Saccharomyces cerevisiae L. (Hope and Struhl, 1987; Landshulz et al., 1988). The activator domain of 
GCN4 resides in a stretch of acidic amino acids located roughly in the centre of the protein while the $\mathrm{N}$-terminal region serves as a sequence-specific DNA binding domain (Hope and Struhl, 1986; Hope et al., 1988).

Yeast GCN5 is a histone acetyltransferase (HAT), which acetylates the specific lysine residues within the $\mathrm{N}$-terminal tails of the core histones in nucleosomes (Brownell et al., 1996). This reduces the strength of the histone-DNA interaction, and the DNA becomes accessible to transcription machinery, resulting in increased levels of transcription of specific gene products (Kuo et al., 1998; Wang et al., 1998). The domains identified within GCN5 include a C-terminal bromo-domain (Tamkun et al., 1992), which interacts with the histone N-termini (Ornaghi et al., 1999), a central domain responsible for interaction with adaptor protein Ada2 (Alteration/Deficiency in activation), and an N-terminal HAT-domain.

While recombinant GCN5 is able to acetylate free histones, the ability to acetylate nucleosomes is acquired only when the protein is present in multi-component complexes (Sterner and Berger, 2000). Yeast GCN5 has been shown to be a component of at least two distinct multi-protein complexes viz. Ada and SAGA, that possess intrinsic HAT activity due to its presence (Grant et al., 1997; Pollard and Peterson, 1997; Grant et al., 1998). The adapter protein ADA2, also present in these complexes, contains several domains. The most $\mathrm{N}$-terminal region includes a cysteine-rich zinc binding domain (comprising of two zinc fingers with six conserved cysteine and two flanking histidine residues). This region is capable of binding to both GCN5 and the transcriptional activation domain of VP16 (Barlev et al., 1995; Candau et al., 1996). ADA2 can also directly interact with bZip type transcriptional activators like yeast GCN4 to modulate transcription of specific genes (Silverman et al., 1994; Barlev et al., 1995) and is thus thought to furnish a link between the upstream activating sequences and the transcription machinery (Barlev et al., 1995). These findings have been established in yeast and little is known about transcriptional activation in higher eukaryotes.

A large number of transcriptional activators of divergent sequence classes have been identified from plants. One of the best characterised is maize Opaque-2 ( $\mathrm{ZmO} 2)$, a bZip type transcriptional activator expressed during late endosperm development (Gallusci et al., 1994). ZmO2 is involved in the regulation of seed storage protein synthesis, modulating the transcription of $22 \mathrm{kDa}$ zein genes, the b-32 albumin gene and a series of genes encoding more minor proteins playing various cellular roles (Kodrzycki et al., 1989; Schmidt et al., 1990; Lohmer et al., 1991). A number of distinct functional domains have been identified within the $\mathrm{ZmO} 2$ protein. These include a basic region followed by a leucine zipper responsible for DNA-binding and dimerization (Hartings et al., 1989; Aukerman et al., 1991) and an N-terminally located acidic activation domain responsible for transcriptional activation (Schmitz et al., 1997). Previous studies have shown that $\mathrm{ZmO} 2$ transactivates a target promoter fused to different reporters in heterologous cell types such as tobacco protoplasts (Lohmer et al., 1991) and this transient expression system was extensively used to characterise the activation domain in the $\mathrm{ZmO} 2$ protein (Schmitz et al., 1997).

Our past work identified putative transcriptional co-activators, GCN5 and ADA2, from maize. ZmGCN5 is expressed throughout the plant with greatest abundance in tissues containing a high proportion of rapidly dividing cells such as young shoots and endosperm (Bhat et al., 2003) while as ZmADA2 is expressed in all tissues and at all stages of development examined (Riehl et al., 2002; Riehl et al., manuscript in preparation). GST spin-down experiments showed that ZmGCN5 interacts with ZmADA2. Homology searches between ZmGCN5 and other known HAT proteins identified a 112AA central domain putatively responsible for this interaction. However no interaction could be observed between $\mathrm{ZmADA} 2$ and $\mathrm{ZmO} 2$ in vitro using GST spindown experiments (Bhat et al., 2003). In view of the fact that ADA2 is part of multi-protein complexes in yeast (Grant et al., 1997), it is quite possible that this interaction requires a stable coactivator complex and thus may require the presence of additional components. This is supported by the observation that by co-immunoprecipitation, in the presence of nuclear extracts, a specific association between $\mathrm{ZmO} 2$ and $\mathrm{ZmADA} 2$ could be detected (Bhat et al., 2003). However, using this approach, it could not be ascertained whether the interaction observed between ZmADA2 and $\mathrm{ZmO} 2$ is direct or indirect. 
In order to define their role in the cellular context, direct protein-protein interactions were tested between $\mathrm{ZmGCN} 5, \mathrm{ZmADA} 2$ and $\mathrm{ZmO} 2$ using Förster resonance energy transfer (FRET) as a detection method.

FRET is a dipole-dipole resonance interaction between two molecules, separated by $1-10 \mathrm{~nm}$, where one molecule, the "donor", transfers its excited state energy radiationless to the other molecule, the "acceptor", (Gadella et al., 1999; Kenworthy, 2001; Mergny and Maurizot, 2001). FRET occurs when putative interacting proteins fused to donor and acceptor fluorescent dyes physically interact (Immink et al., 2002). Fluorescence spectral imaging microscopy (SPIM), acceptor photobleaching (APB) and fluorescence lifetime imaging microscopy (FLIM) have been widely used for documenting FRET (Bastiaens and Jovin, 1996; Bastiaens et al., 1996; Wouters et al., 1998; Bastiaens and Squire 1999; Kenworthy and Edidin, 1999; Kenworthy et al., 2000; Mas et al., 2000; Shah et al., 2001, 2002; Elangovan et al., 2002; Hanley et al., 2002; Immink et al., 2002; Karpova et al., 2003).

We used the APB method to analyse the interactions between ZmGCN5 and ZmADA2 in planta. Finally we used the novel time-correlated single photon counting FLIM to confirm our observations. Our results demonstrate that ZmGCN5 interacts with ZmADA2 in planta and deletion of 112AA central domain from ZmGCN5 abolishes this interaction. Furthermore we show that maize transcriptional activator, $\mathrm{ZmO} 2$ interacts with ZmADA2 inside the nucleus. Our results also demonstrate that the transactivation of $\mathrm{b}-32$ promoter by $\mathrm{ZmO} 2$ is influenced in the presence of the over-expressed co-activators ZmGCN5 and ZmADA2. These observations support the hypothesis that $\mathrm{ZmO} 2$ recruits the co-activators ZmGCN5 and ZmADA2 to modulate its activity at different promoters depending on the cellular and chromosomal context.

\section{Results}

Co-localisation of ZmGCN5, ZmADA2

and $\mathrm{ZmO} 2$ in living plant cells

Co-localisation studies were carried out by transient gene expression in cowpea protoplasts where cyan fluorescent protein (CFP) and yellow fluorescent protein (YFP) were fused to C-terminal ends of ZmGCN5, mutant $\mathrm{ZmGCN}^{\triangle 313-425}$, ZmADA2 and $\mathrm{ZmO} 2$ (Figure 1). All the proteins under study contain putative or previously characterised nuclear localisation sequences (NLS). ZmGCN5 contains an NLS in the N-terminal region and deletion of this NLS renders the protein cytoplasmic (Bhat et al., 2003) while ZmADA2 contains four putative NLS spread across the whole polypeptide (Riehl et al., 2002; Riehl et al., manuscript in preparation). $\mathrm{ZmO} 2$, on the other hand, contains two NLS, one near the N-terminal region (amino acids 101-135) and another in the basic region (amino acids 223-254; Varagona et al., 1991). Protoplasts were co-transfected with constructs expressing: (a) ZmGCN5-CFP and ZmADA2-

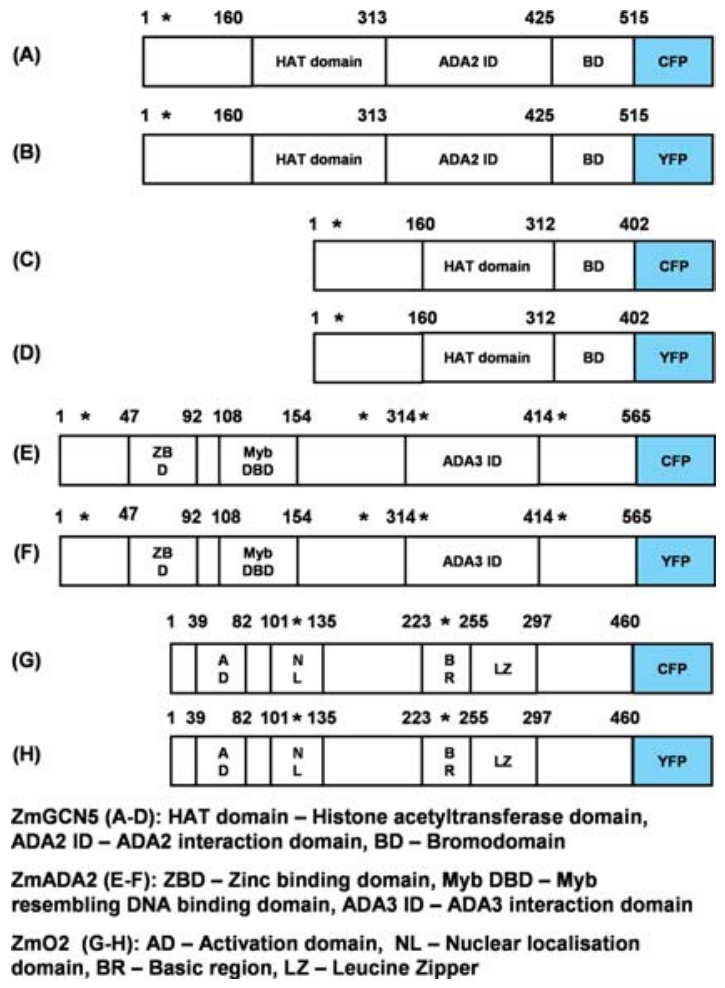

Figure 1. ZmGCN5, ZmADA2 and $\mathrm{ZmO} 2$ constructs used for co-localisation and FRET studies. Domain structures of $\mathrm{ZmGCN}$, ZmADA2 and $\mathrm{ZmO} 2$ are shown as C-terminal fusion proteins with CFP or YFP. A, B. Full length cDNA of ZmGCN5 fused to CFP and YFP genes. C, D. Mutant ZmGCN5 $5^{(\triangle 313-425)}$ fused to CFP and YFP. E, F. Full length cDNA of ZmADA2 fused to CFP and YFP genes. G, H. Full length cDNA of $\mathrm{ZmO} 2$ fused to $\mathrm{CFP} / \mathrm{YFP}$. The asterisks mark the positions of nuclear localisation sequences in the proteins used in the study. 
YFP; (b) mutant $\mathrm{ZmGCN}^{\triangle 313-425}$-CFP, ZmADA2-YFP; (c) ZmO2-CFP and ZmADA2YFP fusion proteins and analysed by confocal laser scanning microscopy (CLSM) after incubation for 18-24 hours (Figure 2).

Figure 2A-D shows the co-localisation of ZmGCN5-CFP and ZmADA2-YFP. From the combined overlay images (Figure 2D) it is clear that the targeting and the localisation pattern of ZmGCN5 and ZmADA2 overlap inside the nucleus. In the case of mutant $\mathrm{ZmGCN}^{\triangle 313-425}$ CFP and ZmADA2-YFP, again both proteins are correctly targeted into the nucleus (Figure 2E and F). As the deleted version of ZmGCN5 should abolish any possible interaction with ZmADA2, this suggests that both proteins employ their own NLS sequences although the nuclear transport of ZmADA2 by association with endogenous ZmGCN5 cannot be ruled out.

An overlay image for $\mathrm{ZmO} 2$ and $\mathrm{ZmADA} 2$ (Figure 2I-H) also shows considerable overlap inside the nucleus. For all the co-localisation combinations, no differences were obtained between CFP and YFP tagged fusion proteins.
FRET-APB studies on interaction between $\mathrm{ZmGCN}$, ZmADA2 and $\mathrm{ZmO2}$

Although the co-localisations of the ZmGCN5, $\mathrm{ZmADA} 2$ and $\mathrm{ZmO} 2$ confirm their close proximity $(>200 \mathrm{~nm})$ and potential for interaction, this was expected as all of them contain NLS sequences. In order to directly study the physical interaction between them, the CFP and YFP fusion proteins were used as donor-acceptor pairs in FRET studies. FRET acceptor photobleaching was used as an initial method to confirm the molecular proximity between the putative interacting proteins. Donor fluorescence increases when the acceptor is photobleached in cells expressing putative interacting proteins fused to donor and acceptor (Karpova et al., 2003). Protoplasts were co-transfected with ZmGCN5-CFP and $\mathrm{ZmADA} 2-Y F P$. Images of a representative protoplast before and after bleaching are shown in Figure3A. With the bleaching of ZmADA2-YFP fluorophore, there was a sharp and sudden increase in the intensity of ZmGCN5-CFP (Figure $3 \mathrm{~A}$ and $\mathrm{B}$, compare panels a and $\mathrm{d}$ in Figure $3 \mathrm{~A}$ ).
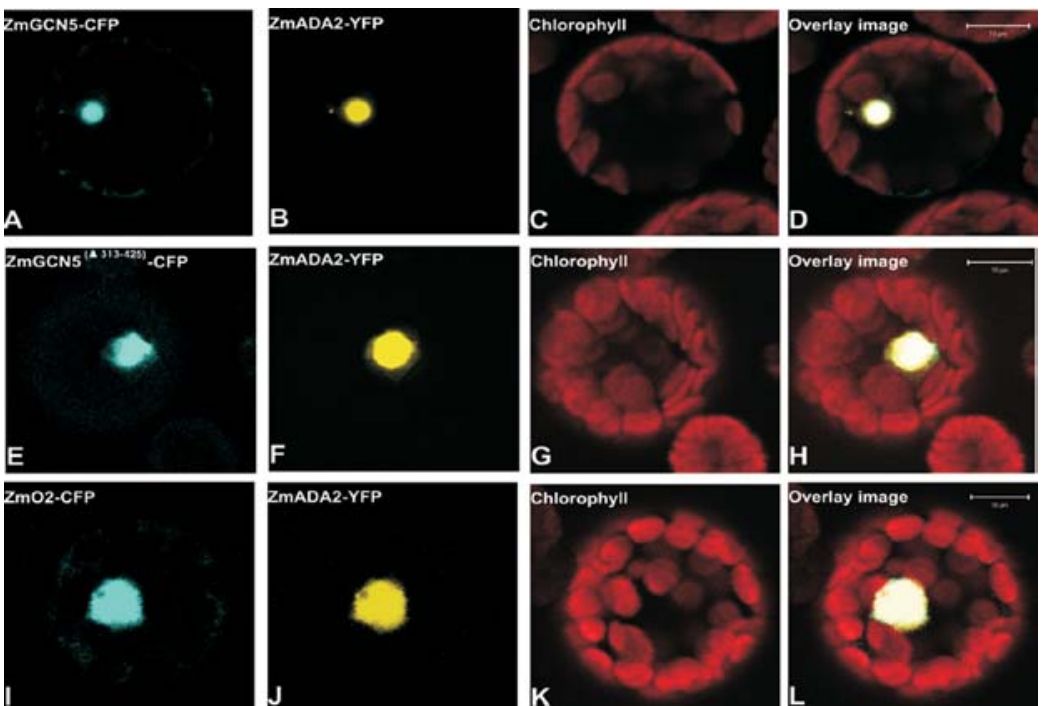

Figure 2. Co-localisation of $\mathrm{ZmGCN} 5, \mathrm{ZmADA} 2$ and $\mathrm{ZmO} 2$ in living plant cells imaged by CLSM A-D. Images of a protoplast coexpressing ZmGCN5-CFP and ZmADA2-YFP. The two fluorophores, CFP and YFP, were simultaneously visualised. A. Nuclear localised ZmGCN5-CFP in cyan. B. Nuclear localised ZmADA2-YFP in yellow. C. Chlorophyll autofluorescence in red. D. Superimposed images of A, B and C showing co-localisation of ZmGCN5-CFP and ZmADA2-YFP in yellowish white colour. E-H. Images of a protoplast co-expressing ZmGCN5 ${ }^{(\triangle 313-425)}-\mathrm{CFP}$ and ZmADA2-YFP. E. Nuclear localised ZmGCN5 $5^{(\triangle 313-425)}-\mathrm{CFP}$. F. Nuclear localised ZmADA2-YFP. G. Chlorophyll autoflorescence. H. Co-localisation of ZmGCN5 ${ }^{\text {(ada2) }}$ CFP and ZmADA2-YFP in yellowish white colour. I-L. Images of a protoplast co-expressing ZmO2-CFP and ZmADA2-YFP. E. Nuclear localised ZmO2-CFP. F. Nuclear localised ZmADA2-YFP. G. Chlorophyll autofluorescence. H. Co-localisation of ZmO2-CFP and ZmADA2-YFP in yellowish white colour. 
(A)

Before photobleaching of ZmADA2-YFP
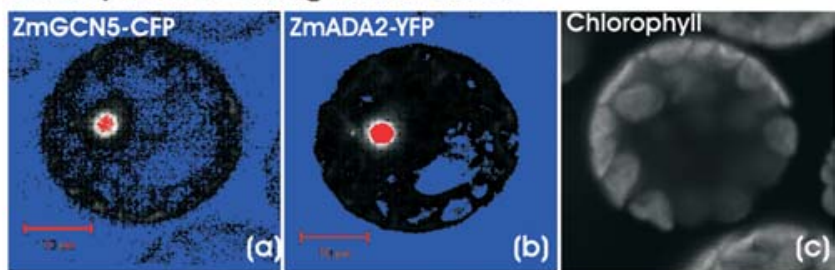

After photobleaching of ZmADA2-YFP
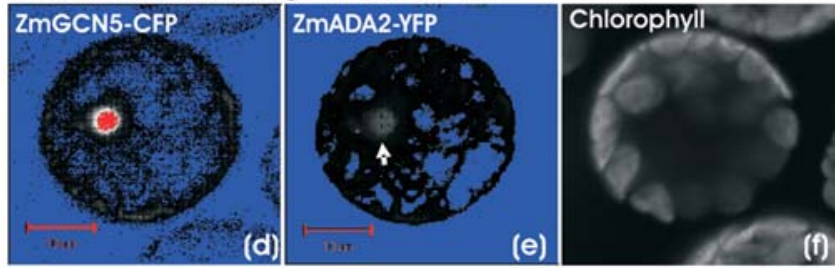

(B)

$\rightarrow$ YFP intensity - - CFP Intensity

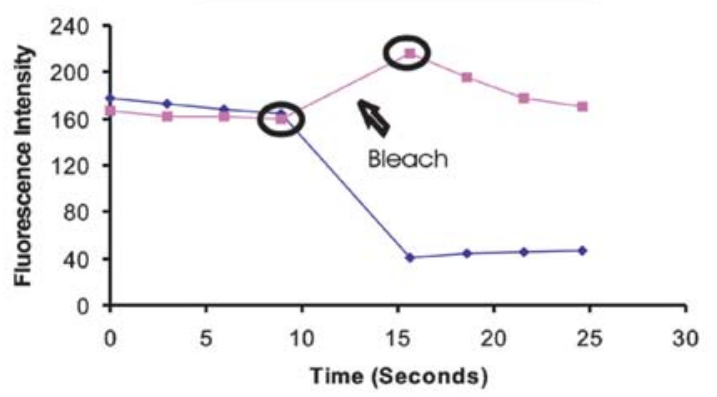

Figure 3. A. Acceptor photobleaching analysis on a cell co-expressing ZmGCN5-CFP and ZmADA2-YFP. a-c. Images before photobleaching. A. Nuclear localised ZmGCN5-CFP b. Same protoplast expressing ZmADA2-YFP c. Chlorophyll autofluorescence. $\mathrm{d}$-f. Images of the same protoplast after photobleaching. d. After the bleach, ZmGCN5-CFP fluorescence increases in the nucleus. e. Photobleached ZmADA2-YFP. Arrow head shows the position of photobleached ZmADA2-YFP. f. Chlorophyll autofluorescence. The images are shown in pseudocolour to highlight the changes in fluorescence intensities. (Blue: no fluorescence, white: low fluorescence and red: high fluorescence. B. Quantification of the donor and the acceptor fluorescence intensity at different time points before and after APB. Some bleaching due to imaging occurs at all the time points except one after the actual photobleaching (shown by arrow) where a substantial increase in the CFP is detected following the YFP bleach. The black circles show the start and end of the bleach cycle.

FRET efficiency, $\mathrm{E}_{\mathrm{F}}$, and background FRET, $\mathrm{B}_{\mathrm{F}}$, was calculated as described in materials and methods. $E_{F}$ values varied from position to position within the same nucleus and also from cell to cell. The $\mathrm{E}_{\mathrm{F}}$ values from one nucleus were pooled to get an average FRET value for one cell in question. Overall APB showed a positive trend and the FRET efficiency in different samples was between $7 \%$ and $38 \%$ (data not shown). FRETAPB experiments, on protoplasts co-expressing fluorescently tagged mutant $\mathrm{ZmGCN5} 5^{\triangle 313-425}$ and ZmADA2, showed no appreciable change in CFP fluorescence after the APB (Figure 4). FRET-APB between fluorescently tagged $\mathrm{ZmO} 2$ and ZmADA2 showed a substantial increase in CFP fluorescence after bleaching the YFP and FRET efficiencies ranged from $8 \%$ to $25 \%$ (data not shown).

In order to get the overall picture for the physical interaction between ZmGCN5, ZmADA2 and $\mathrm{ZmO} 2$, mean FRET efficiencies were calculated for all the interactions tested (Figure 4). Overall the FRET efficiency between ZmGCN5 and $\mathrm{ZmADA} 2$ is around $18.3 \pm 6.86$. This reduces to $-1.45 \pm 4.88$ in case of FRET between mutant $\mathrm{ZmGCN}^{\triangle 313-425}$ and ZmADA2. The mean FRET efficiency between $\mathrm{ZmADA} 2$ and $\mathrm{ZmO} 2$ is $14.26 \pm 4.65$. As a control we did APB in cells coexpressing CFP and YFP, without fusion to any other protein, and the mean random FRET 


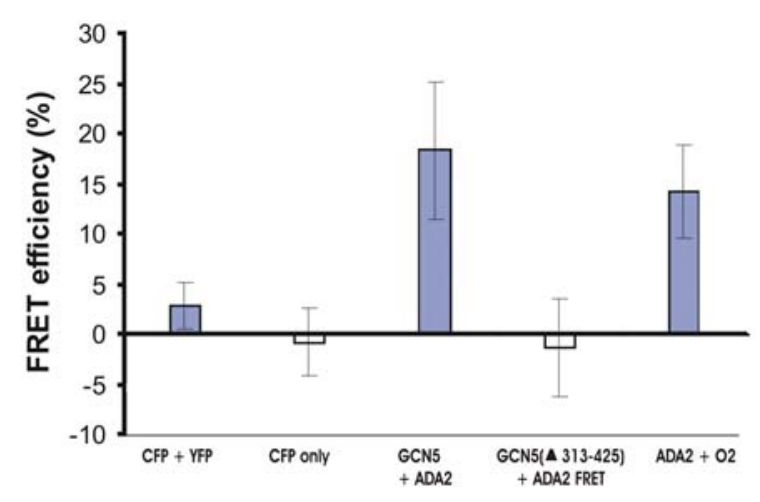

Figure 4. Overall mean FRET efficiency between ZmGCN5, ZmADA2 and ZmO2. ZmGCN5 and ZmADA2 show an overall FRET efficiency of about $18 \%$ which is reduced to almost background levels when the central domain of ZmGCN5 between AA 313 and 425 is deleted. Mean FRET efficiency between $\mathrm{ZmO} 2$ and $\mathrm{Zm}-\mathrm{ADA} 2$ is around $14 \%$. Random FRET efficiency between CFP and YFP (around $2.7 \%$ ) or mock FRET (CFP alone, $0 \%$ ) is significantly less.

efficiency was $2.7 \pm 2.35$. Mock APB-FRET efficiency, in cells expressing CFP alone, was $-0.877 \pm 3.321$.

Taken together, the above results show that the ZmADA2 physically interacts with ZmGCN5 and $\mathrm{ZmO} 2$ within the nucleus.

\section{FRET-FLIM measurements on interaction between $\mathrm{ZmGCN5}, \mathrm{ZmADA} 2$ and $\mathrm{ZmO2}$}

As a final confirmation of the interaction between ZmGCN5, ZmADA2 and $\mathrm{ZmO} 2$, spatially resolved FLIM measurements were done. FRETFLIM is a method to study direct protein-protein interactions by monitoring the fluorescence lifetime of the donor (CFP) molecule. Upon interaction with an acceptor molecule, the donor fluorescence lifetime will decrease. The advantage of this approach is the independence on local fluorophore concentration with no spectral cross talk or photo-bleaching. Fluorescence lifetimes were determined using the Becker \& Hickl SPC 830 module, which is based on the time-correlated single photon counting principle (TCSPC); Becker et al., 2003). Different combinations of ZmGCN5, $\mathrm{ZmADA} 2$ and $\mathrm{ZmO} 2$ were transfected into cowpea protoplasts and the donor lifetimes (CFP) were determined as described in methods.
Figure $5 \mathrm{~A}-\mathrm{F}$ shows an overview of intensity and lifetime images of different combinations of single and double transfected protoplasts. In all images, the fluorescence mainly originates from the nucleus (see also Figure 2).

Nuclei of protoplasts transfected with ZmADA2-CFP alone displayed a mono-exponential decay with an average fluorescence lifetime of $2.5 \mathrm{~ns}$ (Figure 5B). Fluorescence lifetime images of the protoplasts co-transfected with ZmADA2-CFP and ZmGCN5-YFP were analysed according to a bi-exponential fluorescence decay model, in which one component was fixed to the value of $\mathrm{ZmAD}$ A2-CFP alone $(2.5 \mathrm{~ns})$. A significant reduction in the fluorescence lifetime of the donor (ZmADA2CFP) from 2.5 to $1.9-2.0 \mathrm{~ns}$ indicates that both transcriptional co-activators interact (FRET efficiency $20 \%$ ). The same results were obtained for the combination of $\mathrm{ZmADA} 2-\mathrm{CFP}$ and $\mathrm{ZmO} 2-$ YFP, but the energy transfer efficiency was low, as revealed by fluorescence lifetimes of CFP longer than $2.0 \mathrm{~ns}$ in $50 \%$ protoplasts analysed (data not shown). These observations confirm the results obtained from APB experiments (see Figure 4).

The spatial distribution of the fluorescence lifetimes of ZmADA2-CFP in combination with ZmGCN5-YFP is displayed in Figure 5D in pseudo-colours. The image represents the distribution of the fluorescence lifetime in pseudo-colour from blue (2.5 ns: no interaction) to light yellowish-green (1.9-2.0 ns: interaction). In the fluorescence intensity image (Figure 5C) the distribution of the ZmADA2-CFP + ZmGCN5YFP is not equal throughout the nucleus, being stronger in the centre than at the borders. The fluorescence lifetime image (Figure 5D) gives an entirely different picture compared with the fluorescence intensity image. Around the borders, where CFP fluorescence intensity is less, a high incidence of energy transfer could be expected, but the pseudo-colour image for the fluorescence lifetime indicates hardly any interaction. On the contrary the bright fluorescence spot in the centre of the nucleus displays two populations of fluorescence lifetimes. One area (just above the nucleolus) reflects low or no interaction (blue pseudo-colour); while in area around the nucleolus the pseudo-colour indicates an interaction (yellowish-green area). Similar results were obtained when FLIM experiments were repeated on different nuclei. 

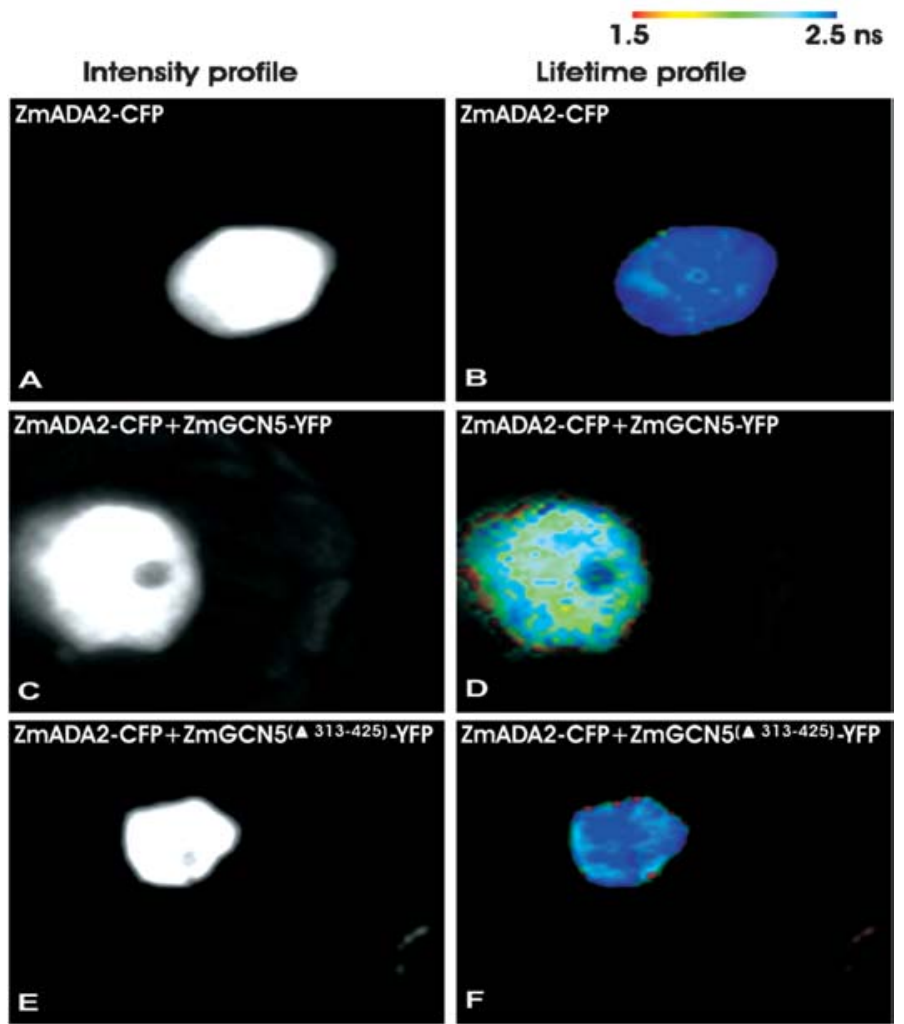

Figure 5. Spatially resolved FLIM analysis on protoplasts co-expressing ZmGCN5 and ZmADA2 fused to CFP and YFP A-F. Intensity and lifetime images within nucleus of protoplasts expressing ZmADA2-CFP alone (A and B) or together with ZmGCN5YFP $(\mathrm{C}$ and $\mathrm{D})$ or with $\mathrm{ZmGCN5} 5^{(\triangle 313-425)}$-YFP $(\mathrm{E}$ and F). The interaction between ZmGCN5 and ZmADA2 leads to reduced CFP lifetimes of 1.9-2 ns in different regions within the nucleus (Yellowish green areas in D) while no reduction in CFP lifetimes is seen in protoplasts expressing ZmADA2-CFP alone or in combination with ZmGCN5 ${ }^{(\triangle 313-425)}$-YFP (B and F, respectively, blue lifetime images). The bar at the top of the image displays the scale of fluorescence lifetimes in pseudo colour from blue (2.5 ns, no interaction) to red (1.5 ns, strong interaction).

For control experiments we transfected ZmADA2-CFP alone (Figure 5B) or in combination with the deletion mutant $\mathrm{ZmGCN} 5^{\triangle 313-425}$ YFP (Figure 5E and F). These images were analysed as described for the interacting proteins (ZmADA2-CFP + ZmGCN5-YFP). For both transfections no interaction was observed. The distribution of the lifetime image is the same for the entire nucleus (blue, $2.5 \mathrm{~ns}$ ) independent of the fluorescence intensity (Figure 5B and 5F).

Transactivation of b-32 promoter by $\mathrm{ZmO2}$ in the presence and absence of coactivators $\mathrm{ZmGCN5}$ and $\mathrm{ZmADA2}$

In order to put the above interactions into their biological context, we decided to take advantage of the transactivating property of $\mathrm{ZmO} 2$ on a previously identified target promoter, that of the b32 gene (Lohmer et al., 1991). For this purpose, a transient expression system in tobacco mesophyll protoplasts was employed (Schmitz et al., 1997). The b-32 promoter is highly responsive to expression in trans of $\mathrm{ZmO} 2$ protein in this assay and thus presented us with a very sensitive method to look at the behaviour of $\mathrm{ZmO} 2$ in the presence and absence of the coactivators ZmGCN5 and ZmADA2. ZmO2 in combination with ZmGCN5, deletion mutant $\mathrm{ZmGCN} 5^{\triangle 313-425}$, or ZmADA2, all expressed from the CaMV 35S promoter, were used to test their ability to transactivate a reporter plasmid, consisting of b-32 promoter fused to $\beta$-glucuronidase (GUS), upon co-transfection. GUS activity was measured $17 \mathrm{~h}$ after co-transfection as described (Schmitz et al., 1997). The results are shown in Figure 6. 


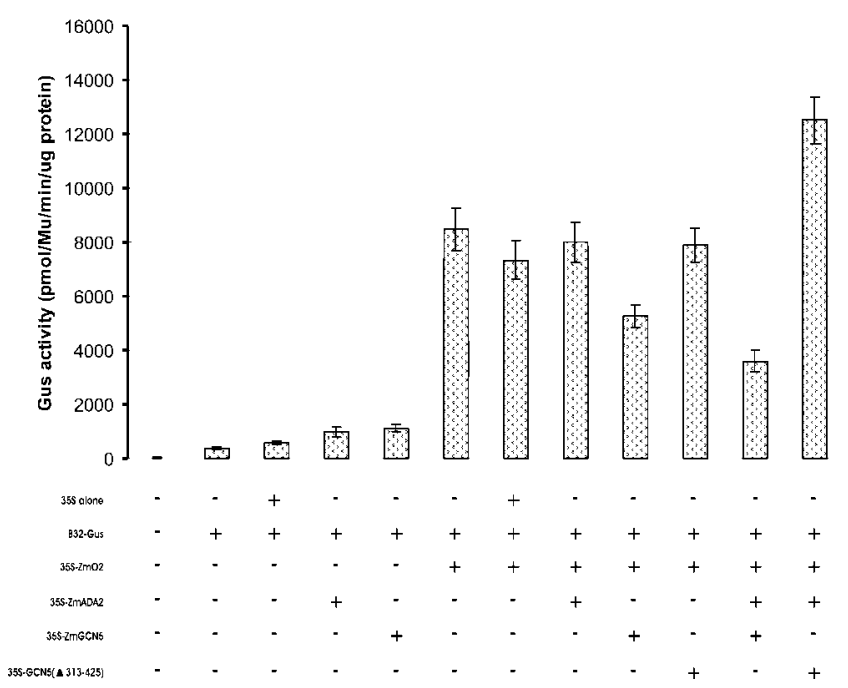

Figure 6. Transactivation of b32-GUS by $35 \mathrm{~S}-\mathrm{ZmO} 2$ in the presence and absence of co-activators $\mathrm{ZmGCN} 5$ and ZmADA2. The reporter plasmid encoding the GUS gene driven by b-32 promoter was co-transfected into the tobacco protoplasts along with different combinations of $35 \mathrm{~S}$ promoter driven $\mathrm{ZmO} 2, \mathrm{ZmGCN} 5, \mathrm{ZmGCN}^{(\triangle 313-425)}$, and ZmADA2. Addition of ZmGCN5 leads to reduction in the measured GUS activity while this inhibitory effect is not seen when the deleted version of ZmGCN5 defective in binding ZmADA2 is used in the transactivation experiments.

Transfection of the reporter plasmid alone, or in combination with expression plasmid, (35S alone), resulted in weak basal level GUS activity. Addition of $35 \mathrm{~S}-\mathrm{ZmGCN} 5$ or $35 \mathrm{~S}-\mathrm{ZmADA} 2$ along with b-32-GUS did not change the GUS levels significantly above the background while cotransfection of b-32-Gus and 35S-ZmO2 stimulated the GUS activity strongly and significantly. Addition of expression plasmid or 35SZmADA2 along with b-32-GUS and 35S-ZmO2 did not change the GUS levels appreciably and they remained the same as the levels obtained by cotransfecting protoplasts with b-32-GUS and 35S-ZmO2. However, surprisingly, cotransfection of protoplasts with b-32-GUS and $35 \mathrm{~S}-\mathrm{ZmO} 2$ in the presence of $35 \mathrm{~S}-\mathrm{ZmGCN} 5$ alone or in combination with $35 \mathrm{~S}-\mathrm{ZmADA} 2$ led to a significant decrease in the measured GUS activity. This inhibitory effect of $35 \mathrm{~S}-\mathrm{ZmGCN} 5$ on the transactivation ability of $\mathrm{ZmO} 2$ was overcome when the deletion mutant of ZmGCN5 (viz. ZmGCN $5^{\triangle 313-425}$, defective in binding ZmADA2 (as reported in this paper), was used for cotransfection instead of the full length protein. Furthermore in the background of the dominant negative ZmGCN5 deletion mutant, addition of full length 35S-ZmADA2 enhanced the transactivation of b-32-GUS by $35 \mathrm{~S}-\mathrm{ZmO} 2$ significantly.
The experiments were repeated several times and the same results were obtained.

\section{Discussion}

\section{Role of histone acetylation in transcriptional} activation

Packaging of the DNA in a eukaryotic cell in the form of chromatin is a highly regulated process with a major influence on the levels of transcription of individual genes (Sterner and Berger, 2000). Certain protein complexes can modify chromatin structure with resultant effects on gene expression (Graessle et al., 2001). A well known mechanism by which chromatin structure can be altered is through the reversible acetylation of lysines within the amino-terminal tails of core histones (Ricci et al., 2002). Enhanced gene expression has often been linked to increased acetylation of nucleosomes (Grunstein, 1997; Struhl, 1998); however there are reports where histone acetylation appears to remain unchanged upon gene activation (CraneRobinson et al., 1997; O`Neil and Turner, 1995). Studies have also been reported where increased acetylation correlates with repression rather than activation (Bartsch et al., 1996; Montecino 
et al., 1999; Deckert and Struhl, 2001; Sheldon et al., 2001; Ricci et al., 2002). GCN5 histone acetyltransferase has been implicated in transcriptional modulation in higher eukaryotes (Sterner and Berger, 2000). Many transcriptional activators are thought to recruit the GCN5-ADA2 complex to effect gene activation (Swanson et al., 2003). In plants, there is evidence for in vitro interactions between $\mathrm{ZmGCN} 5$ and ZmADA2 (maize) and AtGCN5 and AtADA2 (Arabidopsis). ZmO2 co-immunoprecipitates with ZmADA2 only in the presence of nuclear extracts (Bhat et al., 2003), but AtADA2 interacts directly with an acidic transcriptional activator, AtCBF1 (Stockinger et al., 2001). The caveats associated with the results from yeast 2-hybrid interactions and pull-down assays render essential confirmation of these interactions by in planta assays such as those presented here.

Microscopy-based methods such as FRET (Gadella et al., 1999; Miyawaki and Tsien, 2000) allow a sub-cellular resolution of the sites of interaction within the cell. Although there has been widespread interest in this approach, reports of successful FRET are still limited (Karpova et al., 2003). In the present study we used FRET-based methods to directly dissect the interaction of nuclear co-activators GCN5 and ADA2 with a plant transcription factor. As a functional readout of these interactions we used the transactivation ability of $\mathrm{ZmO} 2$ to modulate b-32 promoter driven GUS activity in a robust and reliable transient overexpression system.

\section{ZmGCN5 interacts with ZmADA2 in vivo}

Recombinant ZmGCN5 is able to acetylate only free histones but not the histones within nucleosomes (Bhat et al., 2003). The ability to acetylate nucleosomes is acquired when the acetylase is in the form of multi-protein complexes (Sterner and Berger, 2000). Yeast GCN5 is linked to the SAGA and Ada complexes by its interaction with adaptor protein ADA2. In order to investigate the possible in vivo interactions between the ZmGCN5 and ZmADA2, FRET microscopy was used as a detection method. Co-transfection of protoplasts with ZmGCN5 fused to CFP and ZmADA2 fused to YFP, or vice versa, resulted in the tight co-localisation of both proteins inside the nucleus (Figure 2A-D) implying that any interaction between these two proteins presumably occurs inside the nucleus.
SPIM has successfully been used to detect protein-protein interactions in living cells (Shah et al., 2001, 2002; Immink et al., 2002). However, the method is sensitive for concentration effects and requires equal co-expression of donor and acceptor fusion proteins in a living cell. Although equal amounts of tagged proteins were used in the study, different cells will hardly ever express the same amount of protein (Berney and Danuser, 2003). Secondly in plant cells there is a possibility of the absorption of the donor fluorescence by chlorophyll (Immink et al., 2002) and this can lead to false higher YFP/CFP ratios. In order to circumvent these errors we decided to use APB and FLIM as methods of establishing FRET between our putative interacting proteins. When the YFP fluorophore coupled to ZmADA2 was photobleached so as to make it unavailable for accepting energy from CFP, there was a reproducible increase in fluorescence emitted by the CFP fluorophore coupled to ZmGCN5 (Figure 3 and 4), thus confirming the physical interaction between ZmGCN5 and the adaptor ZmADA2. Deletion of the ADA2 interaction domain in ZmGCN5 did not have any effect on the co-localisation of the two proteins and both were correctly targeted to the nucleus (Figure 2E-H). However, the FRET efficiency between the two proteins dropped from $18.3 \pm 6.8 \%$ (in wild type ZmGCN5 and $\mathrm{ZmADA} 2$ interaction) to $-1.45 \pm 4.88 \%$ (in the mutant $\mathrm{ZmGCN} 5^{\triangle 313-425}$ and ZmADA2 interaction) (Figure 4). FLIM measurements confirmed the above results. The mean lifetime of the donor CFP (fused to ZmGCN5 or ZmADA2) was reduced from $2.5 \mathrm{~ns}$ when expressed alone, to 1.9$2.0 \mathrm{~ns}$ when expressed in presence of the acceptor YFP (fused to ZmADA2 or $\mathrm{ZmGCN5}$, respectively) demonstrating the FRET between CFP and YFP (Figure 5D). However, there was no change in the CFP lifetime when FLIM measurements were done on protoplasts co-expressing mutant $\mathrm{ZmGCN}^{\triangle 313-425}$ and ZmADA2 fused to donor and acceptor. Spatially resolved FRET-FLIM analysis indicated that the interaction between $\mathrm{ZmGCN} 5$ and $\mathrm{ZmADA} 2$ is not uniform inside the nucleus, but displays strong and weak patches. As FRET is strongly a distance dependent phenomenon, this suggests that the interaction between ZmGCN5 and ZmADA2 is highly dynamic. It would be of interest to follow the changes in FRET signatures between these proteins at 
different developmental stages in intact tissues. This, in combination with high resolution microscopy, would perhaps allow the FRETFLIM technique to be used for pinpointing sites of gene activation within the nucleus. Overall our results strongly suggest that $\mathrm{ZmGCN5}$ interacts with $\mathrm{ZmADA} 2$ in planta, and that the interaction exclusively occurs inside the nucleus.

\section{FRET identifies a specific interaction between} $\mathrm{ZmADA} 2$ and the transcriptional activator $\mathrm{ZmO2}$

The biological role of GCN5-containing complexes may be difficult to establish from gene knockout phenotypes, for example, if these are lethal, or if there is redundancy in functional copies of the coding sequence (Bhat et al., 2003). In order to establish a role for GCN5 in maize, its interaction with an endosperm-specific plant transcriptional activator, Opaque-2, was investigated. Most previous reports indicated ADA2 rather than GCN5 to be the co-activator component that interacts directly with the activation domain of transcriptional activators (Barlev et al., 1995).

GST spin-down experiments failed to show an interaction between $\mathrm{ZmO} 2$ and the adaptor ZmADA2 (Bhat et al., 2003). Since ADA2 is part of a multi-protein complex in yeast (Grant et al., 1998) and probably in all higher eukaryotes, it is quite likely that an in vivo-constituted coactivator complex is required to stabilise this interaction. In order to address this possibility we endeavoured to test this interaction by in vivo FRET analysis. Co-transfection of protoplasts with $\mathrm{ZmO} 2$ and ZmADA2 fused to CFP and YFP, respectively (or vice versa) resulted in the localisation of both proteins inside the nucleus (Figure 2I-L). Acceptor photobleaching confirmed the energy transfer between $\mathrm{ZmADA} 2$ and $\mathrm{ZmO} 2$ (Figure 4). FLIM measurements on this interaction pair detected reduced lifetimes of $\sim 2.0 \mathrm{~ns}$ for the donor CFP (fused to either ZmADA2 or ZmO2) when expressed in combination with the acceptor YFP (fused to $\mathrm{ZmO} 2$ or $\mathrm{ZmADA} 2$ ). However, the life time reduction was generally less than that seen for the interaction between $\mathrm{ZmGCN} 5$ and ZmADA2 (data not shown). Since FRET is strongly a distance related phenomenon, the longer CFP life times for this interaction suggest that the molecular distance between $\mathrm{ZmO} 2$ and $\mathrm{ZmADA} 2$ is larger than that between ZmADA2 and ZmGCN5. A possible explanation for relatively low FRET efficiency between $\mathrm{ZmADA} 2$ and $\mathrm{ZmO} 2$ could be that since GCN5-based co-activator complexes are needed at several loci during a limited time-frame within the cell cycle, they may have short halflives.

\section{Transactivation of b-32 promoter by $\mathrm{ZmO} 2$ is} modulated in presence of ZmGCN5 and ZmADA2

FRET-based techniques allowed us to study the molecular interactions between the putative nuclear co-activators and a typical plant transcription factor in great detail. However, they did not reveal any information about the biological significance of these interactions. In order to address this important question, we exploited the transactivation ability of $\mathrm{ZmO} 2$ on $\mathrm{b}-32$ promoter in tobacco protoplasts. It has previously been established that the tobacco mesophyll protoplasts lack the appropriate factors at concentrations which could substitute for the stimulatory effect of $\mathrm{ZmO} 2$ on the transcription of endosperm genes (Lohmer et al., 1991). This presented us with a simple and robust assay in which the effect of ZmGCN5 and/or ZmADA2 on the transcriptional ability of $\mathrm{ZmO} 2$ could be assessed by simply following the changes in the reporter GUS fused to the downstream b-32 promoter.

Co-transfection of protoplasts with b-32 fused to GUS and $35 \mathrm{~S}-\mathrm{ZmO} 2$ stimulated the GUS activity strongly (Figure 6). Transactivation in presence of $35 \mathrm{~S}-\mathrm{ZmADA} 2$ along with $35 \mathrm{~S}-\mathrm{ZmO} 2$ did not increase the GUS activity further. However, GUS activity dropped significantly when 35SZmGCN5 was used in the transactivation assays in addition to $35 \mathrm{~S}-\mathrm{ZmO} 2$ and $35 \mathrm{~S}-\mathrm{ZmADA} 2$. This inhibitory effect of ZmGCN5 was not seen when the deletion mutant of ZmGCN5 (viz. $\mathrm{ZmGCN}^{\triangle 313-425}$ ), defective in ZmADA2-binding, was used instead of the full length protein. Furthermore, in the background of this "dominant negative" ZmGCN5 deletion mutant, addition of full length $35 \mathrm{~S}-\mathrm{ZmADA} 2$ enhanced the transactivation of $\mathrm{b}-32-\mathrm{GUS}$ by $35 \mathrm{~S}-\mathrm{ZmO} 2$ further. One way to rationalise these observations is that there might be a competition between ZmGCN5 and $\mathrm{ZmO} 2$ for binding to the amino terminal of $\mathrm{ZmADA} 2$. In the absence of $\mathrm{ZmGCN5}$, overexpressed $\mathrm{ZmADA} 2$ may bind $\mathrm{ZmO} 2$ preferentially, resulting in higher GUS activity (endogenous 
GCN5 may compete as well and this cannot be ruled out). It may be reversed when full length overexpressed $\mathrm{ZmGCN} 5$ is present assuming the affinity of ZmADA2 for binding ZmGCN5 is greater than that for binding $\mathrm{ZmO} 2$. At present while we have evidence for the $\mathrm{ZmO} / \mathrm{ZmADA} 2$ and $\mathrm{ZmGCN5/ZmADA} 2$ interactions, we have no evidence that all three molecules are in the same complex. It is formally possible that the ZmADA2 molecules that interact with $\mathrm{ZmO} 2$ are a different population to those that interact with ZmGCN5.

On the contrary, however, it is quite possible that $\mathrm{ZmGCN} 5$ represses the transcription mediated by $\mathrm{ZmO} 2$ at the b-32 locus. In this context it would be of interest to generate a non-functional mutant of ZmGCN5 lacking the enzymatic HAT domain but with intact ZmADA2 binding. This would shed more light on the transcriptional modulation by GCN5-ADA2 based coactivator complexes. Experiments are underway to address this question. Furthermore we are also investigating the mutant versions of $\mathrm{ZmO} 2$ that abolish its interaction with the adaptor $\mathrm{ZmADA} 2$ and following the functional consequences of these deletions.

The acidic domain is the most widespread class of activation domain identified, and domain swapping experiments suggest its wide portability. Therefore, the results presented in this study may be taken as a paradigm for other processes of transcriptional modulation in planta involving acidic activation domains.

\section{Materials and methods}

\section{Plasmids}

The entire open reading frame of ZmGCN5 (AJ428540) was amplified with gene specific primers to remove the stop codon and cloned downstream of the $35 \mathrm{~S}$ promoter into the NcoI site of pMON999-CFP or -YFP vectors (CFP, YFP; Shah et al., 2001) using NcoIGCN5Fwd (5'GCGCGGCCATGGACGGCCTCGTGGCG CCGT3') and NcoIGCN5Rev (5'GCGCCCATG GTTGAGAGTTGTGCAAG3') primers. This clone and all subsequently described clones are shown in Figure 1. For the preparation of mutant ZmGCN5, lacking the central putative ADA2 interaction domain, primers pair NcoIGCN5Fwd and HindIIIHATRev (5'GCAAGCTTCAATA
TTCCTCCGTCATAATC $3^{\prime}$ ) and a second pair HindIIIBromoFwd (5'GCAAGCTTGATGCT TGGCCATTCAAAGAG3') and NcoIGCN5Rev were used to amplify the desired ZmGCN5 fragments. The two fragments were ligated using the HindIII site and inserted into the NcoI site of pMON999-CFP/YFP to generate $\mathrm{ZmGCN}^{\triangle 313-425}$.

ZmADA2 (AJ430205) ORF was amplified using NcoIADA2Fwd primer ( $5^{\prime}$ GCCATGGGG CGGTCGCGAGGGGTGC AGAA3') and NcoIADA2Rev primer (5'GCCCATGGCCGTA GGCAACTC CACAT3') and cloned into the pMON999-CFP/YFP vectors. Similarly $\mathrm{ZmO2}$ (AJ491297) was amplified with specific primers ClaIO2Fwd (5'GCATCGATATGGAGCACGT CATCTCAATG $\left.3^{\prime}\right)$ and XbaIO2Rev (5'GCTCT AGAATACATGTCCATGTGTATGGC ${ }^{\prime}$ ) and cloned into the $\mathrm{ClaI} / \mathrm{XbaI}$ digested pMON999$\mathrm{CFP} / \mathrm{YFP}$ vectors.

For the transactivation experiments ZmGCN5, $\mathrm{ZmGCN}^{\triangle 313-425}$ and ZmADA2 were cloned into the 35S-promoter driven pRT 104 vector (Topfer et al., 1988). 35S-ZmO2 and b-32 GUS vectors have been previously described (Lohmer et al., 1991; Schmitz et al., 1997).

\section{Protoplast transfection}

Cowpea (Vigna unguiculata) mesophyll protoplasts were isolated and transfected as described by Shah et al. (2001).

Transactivation experiments in tobacco mesophyll protoplasts

The transient expression of the plasmids in tobacco protoplasts cv. Petit Havana SR1, was performed as described by Negrutiu et al. (1987). The transactivation experiments and the GUS activities were essentially measured as described before (Lohmer et al., 1991; Schmitz et al. 1997).

\section{Co-localisation studies using confocal microscopy}

Detailed analysis of intracellular fluorescence was performed by confocal laser scanning microscopy using a Zeiss LSM 510 microscopy system (CarlZeiss) based on an Axiovert inverted microscope equipped with an Argon ion laser as an excitation source. CFP- and YFP-tagged proteins, expressed in protoplasts, were excited by the $458 \mathrm{~nm}$ and the 
$514 \mathrm{~nm}$ laser lines sequentially. CFP fluorescence was selectively detected by an HFT 458 dichroic mirror and BP 470-500 band pass emission filter while YFP fluorescence was selectively detected by using an HFT 514 dichroic mirror and BP 535-590 band pass emission filter. In both cases the chlorophyll autofluorescence was filtered out and detected in another channel using a LP650 long pass filter. A 25 x Plan-Neofluar water immersable objective lens (N.A 0.8) was used for scanning protoplasts. In order to avoid cross-talk between CFP and YFP, images were acquired in the multichannel tracking mode and analysed with Zeiss LSM510 software.

\section{Acceptor photobleaching (FRET-APB)}

Acceptor photobleaching experiments were performed essentially as described by Kenworthy et al. (2001) and modified by Karpova et al. (2003). Cells were bleached in the acceptor YFP channel by scanning a region of interest (ROI) around the nucleus using 5-20 times $514 \mathrm{~nm}$ argon laser line at $100 \%$ intensity. The bleach time ranged from 5 to $10 \mathrm{sec}$ depending on size of the ROI. Before and after the acceptor bleaching, the CFP intensity images were collected to assess the changes in the donor fluorescence.

FRET efficiency $\left(\mathrm{E}_{\mathrm{F}}\right)$ was calculated using the following formula $E_{F}=\left(I_{5}-I_{4}\right) \times 100 / I_{5}$, where $I_{5}$ is the CFP intensity after the photobleaching of YFP and $\mathrm{I}_{4}$ is the intensity just before the photobleaching. This formula thus yields the increase in CFP fluorescence following a YFP bleach normalised by CFP fluorescence after the bleach (Karpova et al., 2003). In order to monitor the changes in the levels of CFP fluorescence before the bleaching process, background FRET efficiency $\left(\mathrm{B}_{\mathrm{F}}\right)$ was calculated using the following formula $B_{F}=\left(I_{4}-I_{3}\right) \times 100 / I_{4}$, where $I_{3}$ and $I_{4}$ refer to the CFP intensity at time points 3 and 4 preceding the bleaching. In all cases the background was insignificantly low and the background subtractions were not performed.

\section{Fluorescence Lifetime Imaging Microscopy (FRET-FLIM)}

Interaction between a donor (CFP) and an acceptor (YFP) molecule leads to reduction of the fluorescence lifetime of the donor. The amount of reduction is directly correlated with the FRET efficiency via $E=1-\tau_{\mathrm{DA}} / \tau_{\mathrm{D}}$ where $\tau_{\mathrm{DA}}$ is the fluorescence lifetime of the donor (CFP) in the presence of acceptor (YFP) and $\tau_{\mathrm{D}}$ is the fluorescence lifetime of the donor.

FRET-FLIM was performed using a Biorad Radiance 2100 MP system in combination with a Nikon TE 300 inverted microscope. $860 \mathrm{~nm}$ two photon excitation (TPE) was used to excite CFP. TPE pulses were generated by a Ti:Sapphire laser (Coherent Mira) that was pumped by a $5 \mathrm{~W}$ Coherent Verdi laser. Pulse trains of $76 \mathrm{MHz}$ (150 fs pulse duration, $860 \mathrm{~nm}$ center wavelength) were produced. The excitation light was directly coupled into the microscope and focused into the sample using a CFI Plan Apochromat $60 \mathrm{x}$ water immersion objective lens (N.A. 1.2). Fluorescent light was detected using the non-descanned single photon counting detection. For the FLIM experiment the Hamamatsu R3809U MCP PMT was used (time resolution $50 \mathrm{ps}$ ). CFP emission was selected using a $480 \mathrm{DF} 30 \mathrm{~nm}$ band-pass filter. Images with a frame size of $64 \times 64$ pixels were acquired using the Becker and Hickl SPC 830 module (Becker et al., 2003). The average count rate was $2 \times 10^{4}$ photons/s, for an acquisition time of $90 \mathrm{sec}$. From the intensity images, complete fluorescence lifetime decays were calculated per pixel and fitted using a double exponential decay model. The lifetime of one component was fixed to the value found for ZmADA2-CFP (2.5 ns).

\section{Acknowledgements}

The authors wish to thank Brigitte Piegeler for help with the preparation and transfection of tobacco mesophyll protoplasts. RB thanks the Deutsche Forschungsgemeinschaft (DFG) for a $\mathrm{PhD}$ stipendium in the framework of the Graduiertenkolleg Molekulare Analyse von Entwicklungsprozessen (2000-2002).

\section{References}

Aukerman, M.J., Schmidt, R.J., Burr, B. and Burr, F.A. 1991. An arginine to lysine substitution in the bZIP domain of an opaque-2 mutant in maize abolishes specific DNA binding. Genes Dev. 5: 310-320.

Barlev, N.A., Candau, R., Wang, L., Darpino, P., Silverman, N. and Berger, S.L. 1995. Characterization of physical 
interactions of the putative transcriptional adaptor, ADA2, with acidic activation domains and TATA-binding protein. J. Biol. Chem. 270: 19337-19344.

Bartsch, J., Truss, M., Bode, J. and Beato, M. 1996. Moderate increase in histone acetylation activates the mouse mammary tumor virus promoter and remodels its nucleosome structure. Proc. Natl. Acad. Sci. USA. 93: 10741-10746.

Bastiaens, P.I. and Jovin, T.M. 1996. Microspectroscopic imaging tracks the intracellular processing of a signal transduction protein: fluorescent-labeled protein kinase C beta I. Proc. Natl. Acad. Sci. USA. 93: 8407-8412.

Bastiaens, P.I., Majoul, I.V., Verveer, P.J., Soling, H.D. and Jovin, T.M. 1996. Imaging the intracellular trafficking and state of the AB5 quaternary structure of cholera toxin. Embo. J. 15: 4246-4253.

Bastiaens, P.I. and Squire, A. 1999. Fluorescence lifetime imaging microscopy: spatial resolution of biochemical processes in the cell. Trends. Cell. Biol. 9: 48-52.

Becker, W., Bergman, A., Biskup, C, Kelbaukas, L., Zimmer, T., Kloecker, N. and Bendorf, K. 2003. High resolution TCSPC lifetime imaging. Proc. SPIE. 4963: 1-10.

Berger, S.L., Pina, B., Silverman, N., Marcus, G.A., Agapite, J., Regier, J.L., Triezenberg, S.J. and Guarente, L. 1992. Genetic isolation of ADA2: a potential transcriptional adaptor required for function of certain acidic activation domains. Cell. 70: 251-265.

Berney, C. and Danuser, G. 2003. FRET or No FRET: a quantitative comparison. Biophys. J. 84: 3992-4010.

Bhat, R.A., Riehl, M., Santandrea, G., Velasco, R., Slocombe, S., Donn, G., Steinbiss, H.H., Thompson, R.D. and Becker, H.A. 2003. Alteration of GCN5 levels in maize reveals dynamic responses to manipulating histone acetylation. Plant J. 33: 455-469.

Brownell, J.E., Zhou, J., Ranalli, T., Kobayashi, R., Edmondson, D.G., Roth, S.Y. and Allis, C.D. 1996. Tetrahymena histone acetyltransferase A: a homolog to yeast $\operatorname{Gen} 5 \mathrm{p}$ linking histone acetylation to gene activation. Cell. 84: 843-851.

Candau, R. and Berger, S.L. 1996. Structural and functional analysis of yeast putative adaptors. Evidence for an adaptor complex in vivo. J. Biol. Chem. 271: 5237-5245.

Crane-Robinson, C., Hebes, T.R. and Thorne, A.W. 1997. Chromosomal mapping of core histone acetylation by immunoselection. Methods 12: 48-56.

Deckert, J., Struhl, K. 2001. Histone acetylation at promoters is differentially affected by specific activators and repressors. Eur. J. Biochem. 57: 231-239.

Drysdale, C.M., Duenas, E., Jackson, B.M., Reusser, U., Braus, G.H. and Hinnebusch, A.G 1995. The transcriptional activator GCN4 contains multiple activation domains that are critically dependent on hydrophobic amino acids. Mol. Cell. Biol. 15: 1220-12233.

Elangovan, M., Day, R.N. and Periasamy, A. 2002. Nanosecond fluorescence resonance energy transfer-fluorescence lifetime imaging microscopy to localize the protein interactions in a single living cell. J. Microsc. 205: 3-14.

Gadella, T.W.Jr., van der Krogt, G.N. and Bisseling, T. 1999. GFP-based FRET microscopy in living plant cells. Trends Plant Sci. 4: 287-291.

Gallusci, P., Salamini, F. and Thompson, R.D. 1994. Differences in cell type-specific expression of the gene Opaque 2 in maize and transgenic tobacco. Mol. Gen. Genet. 244: 391-400.

Geisberg, J.V., Lee, W.S., Berk, A.J and Ricciardi, R.P. 1994. The zinc finger region of the adenovirus E1A transactivating domain complexes with the TATA box binding protein. Proc. Natl. Acad. Sci. USA 91: 2488-2492.

Graessle, S., Loidl, P. and Brosch, G. 2001. Histone acetylation: plants and fungi as model systems for the investigation of histone deacetylases. Cell Mol. Life Sci. 58: 704-720.

Grant, P.A., Duggan, L., Cote, J., Roberts, S.M., Brownell, J.E., Candau, R., Ohba, R., Owen-Hughes, T., Allis, C.D., Winston, F., Berger, S.L., and Workman, J.L. 1997. Yeast Gen5 functions in two multisubunit complexes to acetylate nucleosomal histones: characterization of an Ada complex and the SAGA (Spt/Ada) complex. Genes Dev. 11: 1640-1650.

Grant, P.A., Sterner, D.E., Duggan, L.J., Workman, J.L. and Berger, S.L. 1998. The SAGA unfolds: convergence of transcription regulators in chromatin-modifying complexes. Trends Cell Biol. 8: 193-197.

Grunstein, M. 1997. Histone acetylation in chromatin structure and transcription. Nature. 389: 349-352.

Guarente, L., Yocum, R.R. and Gifford, P. 1982. A GAL10CYC1 hybrid yeast promoter identifies the GAL4 regulatory region as an upstream site. Proc. Natl. Acad. Sci. USA. 79: 7410-7414.

Hanley, Q.S., Arndt Jovin, D.J. and Jovin, T.M., 2002. Spectrally resolved fluorescence lifetime imaging microscopy. Appl. Spect. 56: 155-166.

Hartings, H., Maddaloni, M., Lazzaroni, N., Di Fonzo, N., Motto, M., Salamini, F. and Thompson, R. 1989. The O2 gene which regulates zein deposition in maize endosperm encodes a protein with structural homologies to transcriptional activators. Embo. J. 8: 2795-2801.

Holstege, F.C., Jennings, E.G., Wyrick, J.J., Lee, T.I., Hengartner, C.J. Green, M.R. Golub, T.R. and Lander, E.S. 1998. Dissecting the regulatory circuitry of a eukaryotic genome. Cell. 95: 717-728.

Hope, I.A., Mahadevan, S. and Struhl, K. 1988. Structural and functional characterization of the short acidic transcriptional activation region of yeast GCN4 protein. Nature 333: 635-640.

Hope, I.A. and Struhl, K. 1987. GCN4, a eukaryotic transcriptional activator protein, binds as a dimer to target DNA. Embo. J. 6: 2781-2784.

Hope, I.A. and Struhl, K. 1986. Functional dissection of a eukaryotic transcriptional activator protein, GCN4 of yeast. Cell 46: 885-894.

Immink, R.G., Gadella, T.W.Jr., Ferrario, S., Busscher, M. and Angenent, G.C. 2002. Analysis of MADS box proteinprotein interactions in living plant cells. Proc. Natl. Acad. Sci. USA 99: 2416-2421.

Karpova, T.S., Baumann, C.T., He, L., Wu, X., Grammer, A., Lipsky, P., Hager, G.L. and McNally, J.G. 2003. Fluorescence resonance energy transfer from cyan to yellow fluorescent protein detected by acceptor photobleaching using confocal microscopy and a single laser. J. Microsc. 209: 56-70.

Kenworthy, A.K. 2001. Imaging protein-protein interactions using fluorescence resonance energy transfer microscopy. Methods 24: 289-96.

Kenworthy, A.K. and Edidin, M. 1999. Imaging fluorescence resonance energy transfer as probe of membrane organiza- 
tion and molecular associations of GPI-anchored proteins. Methods Mol. Biol. 116: 37-49.

Kenworthy, A.K., Petranova, N. and Edidin, M. 2000. Highresolution FRET microscopy of cholera toxin B-subunit and GPI-anchored proteins in cell plasma membranes. Mol. Biol. Cell 11: 1645-1655.

Kodrzycki, R., Boston, R.S. and Larkins, B.A. 1989. The opaque-2 mutation of maize differentially reduces zein gene transcription. Plant Cell 1: 105-114.

Kuo, M.H., Zhou, J., Allis, C.D., and Berger, S.L. 1998. Histone acetyltransferase activity of yeast Gcn $5 p$ is required for the activation of target genes in vivo. Genes Dev. 12: 627-639.

Landschulz, W.H., Johnson, P.F. and McKnight, S.L. 1988. The leucine zipper: a hypothetical structure common to a new class of DNA binding proteins. Science. 240: 1759-1764.

Lin, H., Yutzey, K.E. and Konieczny, S.F. 1991. Musclespecific expression of the troponin I gene requires interactions between helix-loop-helix muscle regulatory factors and ubiquitous transcription factors. Mol. Cell Biol. 11: 267-280.

Lohmer, S., Maddaloni, M., Motto, M., Di Fonzo, N., Hartings, H., Salamini, F. and Thompson, R.D. 1991. The maize regulatory locus Opaque-2 encodes a DNA-binding protein which activates the transcription of the b-32 gene. Embo J. 10: 617-624.

Mas, P., Devlin, P.F., Panda, S. and Kay, S.A. 2000. Functional interaction of phytochrome B and cryptochrome 2. Nature 408: 207-211.

Mergny, J.L. and Maurizot, J.C. 2001. Fluorescence resonance energy transfer as a probe for g-quartet formation by a telomeric repeat. Chembiochem. 2: 124-132.

Miyawaki, A. and Tsien, R.Y. 2000. Monitoring protein conformations and interactions by fluorescence resonance energy transfer between mutants of green fluorescent protein. Methods Enzymol. 327: 472-500.

Montecino, M., Frenkel, B., van Wijnen, A.J., Lian, J.B., Stein, G.S. and Stein, J.L. 1999. Chromatin hyperacetylation abrogates vitamin D-mediated transcriptional upregulation of the tissue-specific osteocalcin gene in vivo. Biochemistry 38 : 1338-1345.

Negrutiu, I., Shillito, R., Potrykus, I., Biasini, G. and Sala, F. 1987. Hybrid genes in the analysis of transformation conditions. Plant Mol. Biol. 8: 363-373.

O'Neill, L.P. and Turner, B.M. 1995. Histone H4 acetylation distinguishes coding regions of the human genome from heterochromatin in a differentiation-dependent but transcription-independent manner. EMBO J. 14: 3646-3957.

Ornaghi, P., Ballario, P., Lena, A.M., Gonzalez, A. and Filetici, P. 1999. The bromodomain of Gen5p interacts in vitro with specific residues in the $\mathrm{N}$ terminus of histone H4. J. Mol. Biol. 287: 1-7.

Pollard, K.J. and Peterson, C.L. 1997. Role for ADA/GCN5 products in antagonizing chromatin-mediated transcriptional repression. Mol. Cell Biol. 17: 6212-6222.

Ricci, A.R., Genereaux, J., and Brandl, B.J. 2002. Components of the SAGA histone acetyltransferase complex are required for repressed transcription of ARG1 in tich medium. Mol. Cell Biol. 22: 4033-4042.

Riehl, M , Dresselhaus, T, McCormick, S, Thompson, R.D. 2002. Maize pollen expresses a truncated version of the transcriptional coactivator Ada2. MNL. 76: 18-19.
Schmidt, R.J., Burr, F.A., Aukerman, M.J. and Burr, B. 1990. Maize regulatory gene opaque- 2 encodes a protein with a 'leucine-zipper' motif that binds to zein DNA. Proc. Natl. Acad. Sci. USA. 87: 46-50.

Schmitz, D., Lohmer, S., Salamini, F. and Thompson, R.D. 1997. The activation domain of the maize transcription factor Opaque-2 resides in a single acidic region. Nucleic Acids Res. 25: 756-763.

Shah, K., Gadella, T.W.Jr., van Erp, H., Hecht, V. and de Vries, S.C. 2001. Subcellular localization and oligomerization of the Arabidopsis thaliana somatic embryogenesis receptor kinase 1 protein. J. Mol. Biol. 309: 641-655.

Shah, K., Russinova, E., Gadella, T.W.Jr., Willemse, J. and de Vries, S.C. 2002. The Arabidopsis kinase-associated protein phosphatase controls internalization of the somatic embryogenesis receptor kinase 1. Genes. Dev. 16: 1707-1720.

Sheldon, L.A., Becker, M., Smith, C.L. 2001. Steroid hormone receptor-mediated histone deacetylation and transcription at the mouse mammary tumor virus promoter. J. Biol. Chem. 35: 32423-32426.

Silverman, N., Agapite, J., Guarente, L. 1994. Yeast ADA2 protein binds to the VP16 protein activation domain and activates transcription. Proc. Natl. Acad. Sci. USA 91: 11665-11668.

Sterner, D.E. and Berger, S.L. 2000. Acetylation of histones and transcription-related factors. Microb. Mol. Biol. Rev. 64: 435-459.

Stockinger, E .J., Mao, Y., Regier, M.K., Triezenberg, S.J. and Thomashow, M.F. 2001. Transcriptional adaptor and histone acetyltransferase proteins in Arabidopsis and their interactions with CBF1, a transcriptional activator involved in cold-regulated gene expression. Nucl. Acids. Res. 29: 1524-1533.

Struhl, K. 1998. Histone acetylation and transcriptional regulatory mechanisms. Genes. Dev. 12: 599-606.

Swanson, M.J., Qiu, H., Sumibcay, L., Krueger, A., Kim, S.J., Natarajan, K., Yoon, S. and Hinnebusch, A.G. 2003. A multiplicity of coactivators is required by Gen4p at individual promoters in vivo. Mol. Cell Biol. 23: 2800-2820.

Tamkun, J.W., Deuring, R., Scott, M.P., Kissinger, M., Pattatucci, A.M., Kaufman,T.C. and Kennison, J.A. 1992. brahma: a regulator of Drosophila homeotic genes structurally related to the yeast transcriptional activator SNF2/ SWI2. Cell 68(3): 561-72.

Topfer, R., Schell, J., Steinbiss, H.H. 1988. Versatile cloning vectors for transient gene expression and direct gene transfer in plant cells. Nucle. Acids Res. 16: 8725.

Varagona, M.J., Schmidt, R.J. and Raikhel, N.V. 1991. Monocot regulatory protein Opaque- 2 is localized in the nucleus of maize endosperm and transformed tobacco plants. Plant Cell 3: 105-113.

Wang, L., Liu, L. and Berger, S.L. 1998. Critical residues for histone acetylation by Gcn5, functioning in Ada and SAGA complexes, are also required for transcriptional function in vivo. Genes Dev. 12: 640-653.

Wouters, F.S., Bastiaens, P.I., Wirtz, K.W. and Jovin, T.M. 1998. FRET microscopy demonstrates molecular association of non-specific lipid transfer protein (nsL-TP) with fatty acid oxidation enzymes in peroxisomes. Embo J. 17: 7179-7189. 